\title{
Modelling and Order of Acoustic Transfer Functions Due to Reflections from Augmented Objects
}

\author{
Martin Kuster ${ }^{1,2}$ and Diemer de Vries ${ }^{1}$ \\ ${ }^{1}$ Laboratory of Acoustical Imaging and Sound Control, Department of Imaging Science and Technology, \\ Faculty of Applied Sciences, Delft University of Technology, 2600 Delft, GA, The Netherlands \\ ${ }^{2}$ Sonic Arts Research Centre, Faculty of Engineering and Physical Sciences, Queen's University Belfast, \\ Belfast, BT7 1NN, UK
}

Received 30 April 2006; Revised 29 September 2006; Accepted 14 October 2006

Recommended by Aki Härmä

It is commonly accepted that the sound reflections from real physical objects are much more complicated than what usually is and can be modelled by room acoustics modelling software. The main reason for this limitation is the level of detail inherent in the physical object in terms of its geometrical and acoustic properties. In the present paper, the complexity of the sound reflections from a corridor wall is investigated by modelling the corresponding acoustic transfer functions at several receiver positions in front of the wall. The complexity for different wall configurations has been examined and the changes have been achieved by altering its acoustic image. The results show that for a homogenous flat wall, the complexity is significant and for a wall including various smaller objects, the complexity is highly dependent on the position of the receiver with respect to the objects.

Copyright $\odot 2007$ M. Kuster and D. de Vries. This is an open access article distributed under the Creative Commons Attribution License, which permits unrestricted use, distribution, and reproduction in any medium, provided the original work is properly cited.

\section{INTRODUCTION}

For the simulation and prediction of the acoustics in enclosed spaces, the geometry and acoustic properties of the enclosing boundaries are the primary parameters. The acoustic properties are represented by the acoustic impedance of the boundary. For a simple shoebox room with perfectly rigid walls (i.e., infinite acoustic impedance), the method of mirror image sources leads to a solution that satisfies the wave equation exactly [1]. In practice, rooms are neither of simple shoebox shape nor are the walls perfectly rigid. Instead, the modelling of all geometric details down to the order of the shortest acoustic wavelength would be required and the acoustic impedances of practical materials are generally complex, frequency-dependent, and nonlocally reacting [2].

One of the primary aims in room acoustics research over the past two to three decades has been the realistic and reliable prediction of room acoustics from a subset of the detailed geometric and acoustic information required theoretically [3-5]. In particular, the scattering of sound from nonsmooth finite-size surfaces, leading to diffuse reflections [6-9] and diffraction [10-12], has been recognised as one, if not the, key contributing factor.
The research presented in the current paper aims to shed some light on the complexity of measured reflected sound from a single wall comprising a number of smaller objects. As an objective parameter, the complexity is measured by the required model order of the acoustic transfer function (ATF) resulting from the reflections/scattering from the wall. The interesting questions to be raised are to what extent is the complexity of the ATF dependent on the structural details of the wall in the immediate vicinity of (i) the receiver in the case of scattering and/or (ii) the specular reflection point for specular reflection? If the receiver is immediately in front of a wall section containing a sound diffusing object, do the more distant homogenous flat sections of the wall affect the complexity of the ATF or is the scattering by the diffusing object the main influence? What is the complexity when the wall is rendered completely flat compared to the complexity of the original wall configuration? The aim is to find partial answers to some of these questions in terms of quantitative objective parameters, a perceptual evaluation is beyond the scope of the paper.

These problems will be studied using the method of acoustic imaging. Whilst the fundamentals of this method have been explained by the authors in [13], the current paper 
is an extension and application of the method to a practical problem and investigates the changes in the ATFs when the acoustic image is altered. It is important for the reader to understand that the alternative procedure for studying reflections from a single wall in different configurations requires free-field conditions, that is, the wall would have to be built and physically altered in, for example, an anechoic chamber. The proposed method requires neither free-field condition nor the physical alterations. Further, the purpose of the current paper is not to quantify the scattering by the reflecting objects, this approach has been presented in [14]. Whilst, similar to the energetic scattering coefficient, some of the results in the paper are also quoted as single figures for the required model order, this serves merely as an example and the method is much more powerful in that it allows one to obtain and study ATFs from single reflecting objects with geometrical and acoustic properties that are close to reality. The method thus includes both the effects of scattering and the frequency-dependent acoustic impedance of the objects. Since the latter are usually smooth functions of frequency, it is anticipated that the scattering has a larger influence on the model order.

This paper is organised as follows. In the following section, a brief description of the measured reflecting object (wall plus objects) and a brief overview of how acoustic image of the object is obtained and augmented are given. In Section 3, the mathematical basis for the transfer function models is outlined. Finally, the numerical results and the discussion thereof are presented in Sections 4 and 5.

\section{ACOUSTIC IMAGING}

The ATFs to be modelled are due to reflections from a corridor wall including smaller objects such as columns, a closet, and an electrical distribution box. A photograph of the corridor wall is shown in Figure 1. In a previous publication [13], it has been shown how an acoustic image of this wall and the smaller objects is obtained by measuring acoustic impulse responses on a planar array and then extrapolating the acoustic pressure and particle velocity to the reflecting objects.

The process of acoustic imaging has a few interesting properties. Firstly, it simply maps the reflections in the acoustic impulse response to the reflecting object, and does thereby retain a significant amount of the acoustic information. Secondly, it is reversible, that is, the acoustic impulse responses can be recovered from the acoustic image (with some loss of acoustic information). This step is referred to as demigration [13]. Thirdly, the acoustic image can be augmented and then be demigrated (removing an object from the acoustic image results in acoustic impulse responses without the reflections from the object after demigration). Since this involves nothing more than simple copy and paste of measured data, the result is expected to be very close to physically altering the reflecting object and remeasuring the acoustic impulse responses, and arguably closer to reality than the synthetic data from room acoustic models.

The loss of acoustic information in the process is caused by the temporal width $\Delta t_{0}$ of the source pulse (due to finite frequency bandwidth). Suppose a reflecting object is

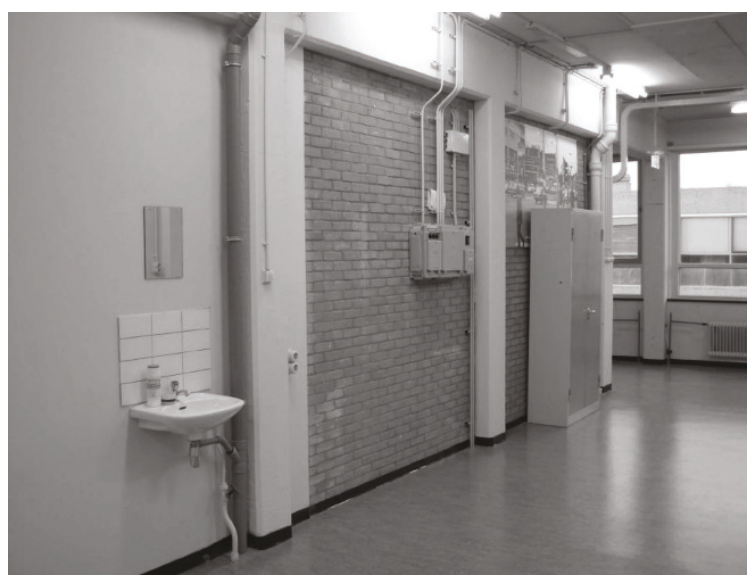

FIgURE 1: Photograph of the corridor wall.

described by a delta function in space. In the corresponding acoustic image, it will appear as an object with approximate width $\Delta t_{0} c$, where $c$ is the speed of sound in air. If two reflecting objects are less than $\Delta t_{0} c$ apart, their corresponding acoustic images overlap, and there are therefore both unresolvable ambiguity and a loss of acoustic information. The losses are quantified by amplitude losses in the demigrated impulse responses [15]. In practice, further losses are caused by the finite aperture of the receiver array and the finite size of the acoustic image. The finite aperture of the receiver array means that not the entire reflected wavefront is captured and the finite size of the acoustic image means that the reflections from objects not present in the acoustic image are missing. In [13], the authors have used a short FIR matching filter to compensate for the difference in magnitude and phase between the originally measured and demigrated impulse responses.

Until now, the authors have not been able to prove the accurateness of the "cut-and-paste" method by comparing it with measurements on the wall with the physical changes. The reason lies in the resources required to perform such measurements under the required free-field conditions (because no other reflections than those from the wall must be present) and matching real and virtual changes exactly could prove to be a challenge, too. Regardless of this issue, the authors would like to stress that the processes of acoustic imaging and demigration have significant similarity with the wellestablished boundary element method and near-field acoustic holography. In particular, they are all derived from the Kirchhoff-Helmholtz or Rayleigh integrals.

\subsection{Signal processing implementation}

In the following, the processes of acoustic imaging and demigration are described explicitly for discrete variables. The position of the single sound source is denoted by $\mathbf{r}_{S}=$ $\left(r_{S_{x}}, r_{S_{y}}, r_{S_{z}}\right)$. The vector $\mathbf{r}_{R}^{[i j]}=\left(r_{R_{x}}^{[i]}, 0, r_{R_{z}}^{[j]}\right)$ denotes the discrete measurement position at the indices $i$ and $j$ in the receiver array (for simplicity, the array is in the $y=0$ plane) 
with spatial sampling intervals $\Delta r_{R_{x}}$ and $\Delta r_{R_{z}}$. The cartesian coordinate vector of pixel $[\mathrm{klm}]$ in the acoustic image is denoted by $\mathbf{r}_{I}^{[\mathrm{klm}]}=\left(r_{I_{x}}^{[k]}, r_{I_{y}}^{[l]}, r_{I_{z}}^{[\mathrm{m}]}\right)$. The value of the time variable $t$ at index $h$ is denoted by $t^{[h]}$.

For discrete temporal and spatial variables, [13, eqaution (2)] for the acoustic image $p_{\operatorname{Im}}$ in terms of reflected pressure has been converted into a summation using piecewise constant integration and then reads

$$
\begin{aligned}
p_{\operatorname{Im}}\left(\mathbf{r}_{I}^{[k l m]}\right)=\sum_{i} \sum_{j} & {\left[\frac{\partial}{\partial t} v_{y}\left(t^{[h]}, \mathbf{r}_{R}^{[i j]}\right) \frac{\rho_{0}}{4 \pi r_{I R}^{[i j k l m]}}\right.} \\
& \left.-\frac{1}{c} \frac{\partial}{\partial t} p\left(t^{[h]}, \mathbf{r}_{R}^{[i j]}\right) \frac{\cos \phi^{[i j k l m]}}{4 \pi r_{I R}^{[i j k l m]}}\right] \Delta r_{R_{x}} \Delta r_{R_{z}} .
\end{aligned}
$$

For notational simplicity, the far-field expression has been used. The mass density and speed of sound in air are $\rho_{0}$ and $c$, respectively, $\cos \phi^{[i j k l m]}=\left|r_{I_{y}}^{[l]}\right| / r_{I R}^{[i j k l m]}$, and the distances $r_{S I}^{[k l m]}$ and $r_{I R}^{[i j k l m]}$ are given by

$$
\begin{gathered}
r_{S I}^{[k l m]}=\sqrt{\left(r_{S_{x}}-r_{I_{x}}^{[k]}\right)^{2}+\left(r_{S_{y}}-r_{I_{y}}^{[l]}\right)^{2}+\left(r_{s_{z}}-r_{I_{z}}^{[m]}\right)^{2}}, \\
r_{I R}^{[i j k l m]}=\sqrt{\left(r_{I_{x}}^{[k]}-r_{R_{x}}^{[i]}\right)^{2}+\left(r_{I_{y}}^{[l]}\right)^{2}+\left(r_{I_{z}}^{[m]}-r_{R_{z}}^{[j]}\right)^{2}} .
\end{gathered}
$$

The time derivatives in (1) can be performed as a preprocessing step by multiplying with $j \omega$ in the frequency domain, with $\omega$ the angular frequency. Equation (1) describes a weighted summation of pressure $p\left(t^{[h]}, \mathbf{r}_{R}^{[i j]}\right)$ and normal component of the particle velocity $v_{y}\left(t^{[h]}, \mathbf{r}_{R}^{[i j]}\right)$ on the array. The time $t^{[h]}$ is given by

$$
t^{[h]}=\frac{r_{S I}^{[k l m]}+r_{I R}^{[i j k l m]}}{c}
$$

and the time index $h$ is therefore a function of both the indices $k, l$, and $m$ of the image point position $\mathbf{r}_{I}$ as well as of the summation indices $i$ and $j$ of the (array) receiver position $\mathbf{r}_{R}$. In practice, the right-hand side of (3) has to be rounded to the nearest integer value of $h$. To minimise this error, measured pressure and normal component of the particle velocity are resampled with a $64 \mathrm{kHz}$ sampling frequency.

The reverse step of recreating the impulse responses from the acoustic image, termed demigration, is given by (7) in [13]. The discretised, far-field approximation reads

$$
p\left(t^{[h]}, \mathbf{r}_{R}^{[i j]}\right)=\sum_{k} \sum_{m} \frac{1}{c} \frac{\partial}{\partial t} p_{\operatorname{Im}}\left(\mathbf{r}_{I}^{[k l m]}\right) \frac{\cos \phi^{[i j k l m]}}{4 \pi r_{I R}^{[i j k l m]}} \Delta r_{I_{x}} \Delta r_{I_{z}},
$$

where $\Delta r_{I_{x}}$ and $\Delta r_{I_{z}}$ are the sampling intervals of the acoustic image in the $x$ - and $z$-direction, and all other variables are defined as above. Since the summand does not depend on time, the time differentiation can only be performed after the summation. The necessary swapping of summation and differentiation order is only permitted for smooth functions. Alternatively, Tygel et al. [16] replace the time derivative and multiplication by $1 / c$ with a space derivative in the $y$-direction,

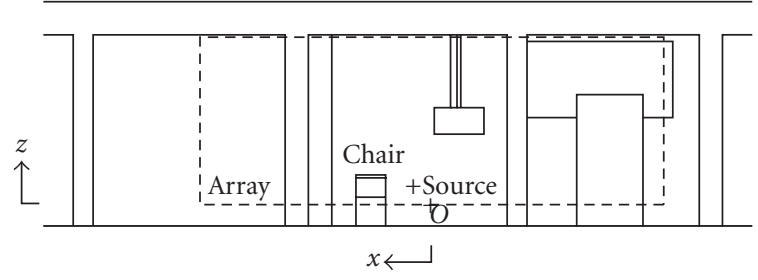

(a)

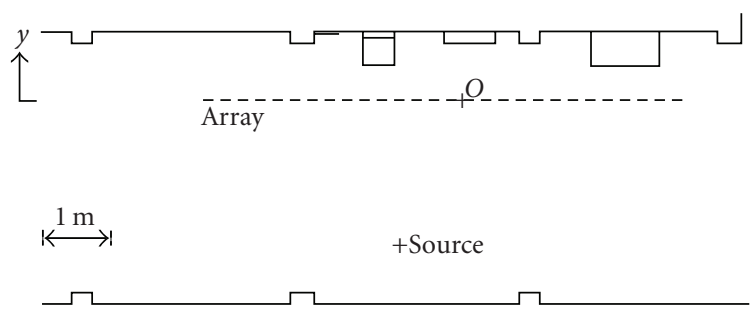

(b)

FIgURE 2: (a) Elevation and (b) floor plan of the corridor with the measurement setup.

which is again a far-field approximation. Equation (4) represents a summation over the acoustic image, where for each value of $t^{[h]}$, the values of $r_{I_{y}}^{[l]}$ are determined by (3). A rounding to the nearest integer value of the index $l$ is required.

For the impulse responses sampled at $64 \mathrm{kHz}$ but the effective bandwidth limited to $8 \mathrm{kHz}$, the coefficients of the matching filter used in [13] are given by

$$
f^{[h]}=[11.3,-23.4,0.5,10.2,0,-8.4,-4.6,1.6,-1.5,0.3] .
$$

Whether the matching filter is a necessity in the current context is debatable, the authors have included it for reasons of consistency.

\subsection{Application to corridor wall and obtaining the ATFs}

A drawing of the measurement setup in front of the corridor wall is shown in Figure 2. The receiver array consists of 140 horizontal and 50 vertical measurement positions and the pressure and normal component of the particle velocity have been measured with a SoundField MKV microphone. With $\Delta r_{R_{x}}=\Delta r_{R_{z}}=0.05 \mathrm{~m}$, the total array aperture is thus $7 \times 2.5 \mathrm{~m}$. The data has been filtered in the wave numberfrequency domain to avoid potential spatial aliasing [17]. A surface representation of the resulting acoustic image of the corridor wall is shown in Figure 3. The sampling in the acoustic image is $\Delta r_{I_{x}}=\Delta r_{I_{y}}=\Delta r_{I_{z}}=0.02 \mathrm{~m}$.

In previous works, the acoustic image has been altered by removing the electrical distribution box [13], replacing the electrical distribution box and the closet with a flat wall section [18] and placing an acoustic diffuser on a flat wall section [14]. The corresponding changes in the impulse responses have been evaluated perceptually and it was found that the presence of the electrical distribution box and closet 


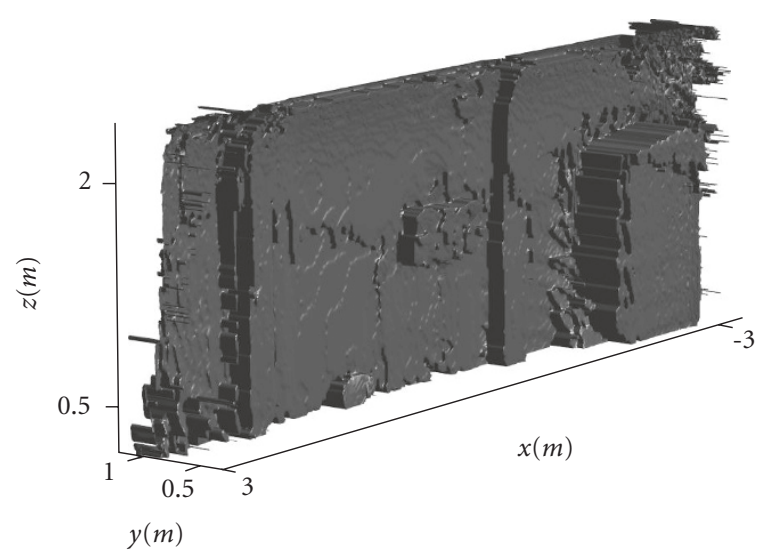

FIGURE 3: Surface presentation of the acoustic image of the corridor wall.

is only audible if the receiver is in their immediate vicinity. The presence of the diffuser was audible even when the ratio of reflected energy between the wall configurations with and without diffuser was almost unity.

For the purpose of the present investigation, the acoustic image of the corridor wall has been augmented and then demigrated in three configurations:

(1) the original unaltered image;

(2) cleaning up the original image by removing the second-order reflection via the floor and the ceiling;

(3) image with completely flat wall obtained by copy/paste of homogenous wall sections, that is, no closet, columns, and so forth.

The reader is reminded that all three configurations including the last one still contain the frequency-dependent acoustic impedance of the wall.

In the following, configuration (3) is referred to as the homogenous flat wall but it needs to be emphasised that this wall is only flat and homogenous on a macroscopic $(>0.05 \mathrm{~m})$ but not microscopic $(<0.05 \mathrm{~m})$ level. A crosssection of the wall therefore can not be characterised by a spatial delta function at a constant position, as would be the case with a mirror image source model, but contains all the local variations associated with an actual brick wall.

The ATFs $H\left(\omega^{[k]}\right)$, that are to be modelled in the next section, are obtained from the demigrated acoustic impulse responses by

$$
H\left(\omega^{[k]}\right)=\operatorname{DFT}\left\{p\left(t^{[h]}, \mathbf{r}_{R}^{[i j]}\right)\right\} .
$$

Because of the antispatial aliasing filter, a temporal sampling frequency of $16 \mathrm{kHz}$ is sufficient, and therefore $\omega_{\max }=$ $50265 \mathrm{rad} / \mathrm{s}$. The DFT was performed with 2048 samples.

Another processing step was to remove the delay corresponding to the travel time of the (approximate) specular reflection path from the source via the reflecting object to the receiver. Whilst the delay does not influence the magnitude

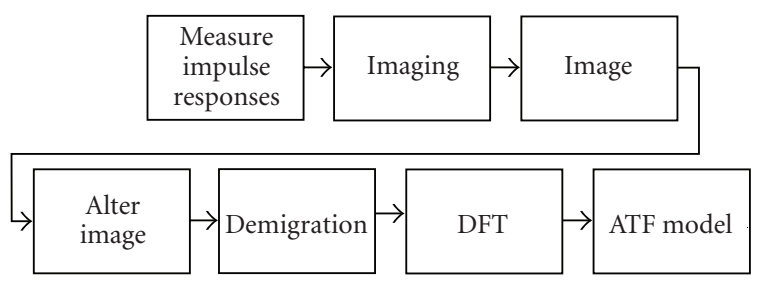

FIGURE 4: Block diagram of the processing from the acoustic image to the ATF model.

response, it does introduce a linear phase shift as a function of frequency, which has to be incorporated into the model and does bias the results for the model order. The delay was removed because it would be present even with a mirror image source model and does not represent any acoustic properties of the reflecting object itself.

A block diagram outlining the required signal processing steps from the impulse responses to the ATF model is given in Figure 4. It is to be emphasised that the ATFs contain neither reflections from other walls in the corridor hall nor the direct sound.

\section{TRANSFER FUNCTION MODEL}

The general model of a transfer function can be written as a rational function of the form

$$
H^{\prime}(s)=\frac{B(s)}{A(s)}=\frac{\sum_{m=0}^{M} b^{[m]} s^{m}}{\sum_{n=0}^{N} a^{[n]} s^{n}},
$$

where $s=j \omega$ and $B$ and $A$ are the polynomials with coefficients $b^{[m]}$ and $a^{[n]}$, respectively.

If $H\left(\omega^{[k]}\right)$ is the ATF to be modelled, the model is obtained from the following equation error:

$$
\min _{b, a} \sum_{k} w\left(\omega^{[k]}\right)\left|H\left(\omega^{[k]}\right) A\left(\omega^{[k]}\right)-B\left(\omega^{[k]}\right)\right|^{2},
$$

where $B\left(\omega^{[k]}\right)$ and $A\left(\omega^{[k]}\right)$ are the values of the numerator and denominator of (7) evaluated at the discrete frequency points $\omega^{[k]}$ determined by the DFT grid points. $w\left(\omega^{[k]}\right)$ is an optional weight function used to give greater emphasis to certain frequencies. Equation (8) results in a system of linear equations in the polynomial coefficients $a^{[n]}$ and $b^{[m]}$ that can be solved by matrix inversion in the least-squares sense $[19,20]$.

The weight function was defined as follows:

$$
w\left(\omega^{[k]}\right)= \begin{cases}0 & \text { for } 0.97 \omega_{\max }<\omega^{[k]}<0.03 \omega_{\max }, \\ 1 & \text { for } 0.97 \omega_{\max } \geq \omega^{[k]} \geq 0.03 \omega_{\max }\end{cases}
$$

The low weight at the extreme ends of the frequency band afford the algorithm a high degree of freedom in that region.

An important and difficult issue is the order selection of the polynomials in the numerator and denominator of the transfer function model. It is well known that an accurate model of a measured room transfer function requires 


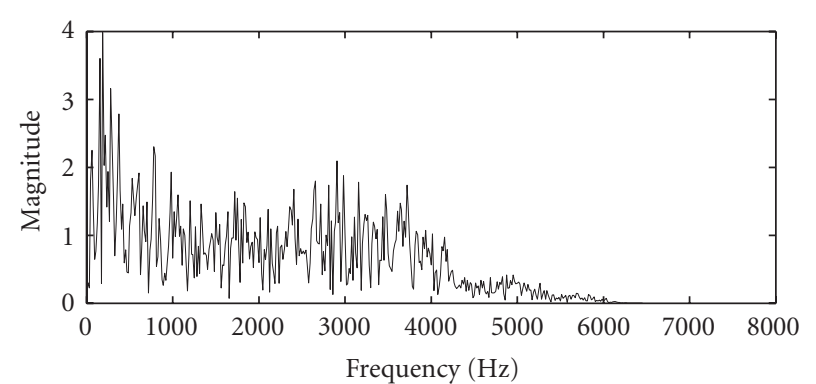

(a)

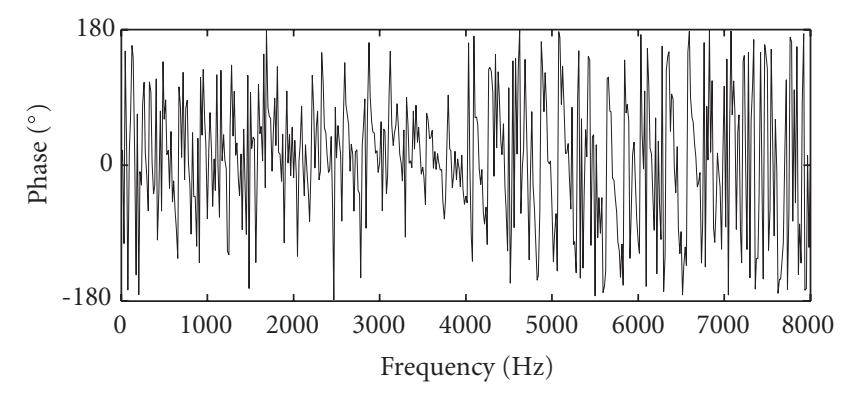

(b)

Figure 5: Typical (a) magnitude and (b) phase of measured room transfer function in the corridor hall.

in excess of a thousand coefficients [21, 22] for both the numerator and denominator. The reasons are the numerous peaks and dips caused by the complex summation of many eigenmodes with quasirandom phases as discovered by Schroeder [23]. As an example, Figure 5 shows the magnitude and phase response of the room transfer function in the corridor hall (not just the single wall!). Since the average frequency spacing between adjacent dips and adjacent peaks is equal [23], it would seem that the order of numerator and denominator polynomials should also be approximately equal at least for the case of the whole room transfer function.

For the purpose of modelling the ATFs from the single augmented wall, the required model orders are much smaller and it proved feasible to model the entire frequency band without resorting to subbands [24]. For reasons of simplification, the order of the numerator and denominator polynomials was kept equal $(N=M)$. Thus, if in the remainder of the paper a model order $N$ is quoted, the actual total order of the model is $N+M=2 N$.

\section{RESULTS}

The following cases for the groups of eleven receiver positions have been investigated:

(A) in front of the closet $(z=0.5 \mathrm{~m})$;

(B) in front of the closet $(z=1.5 \mathrm{~m})$;

(C) in front of the electrical distribution box;

(D) in front of a homogenous wall section;

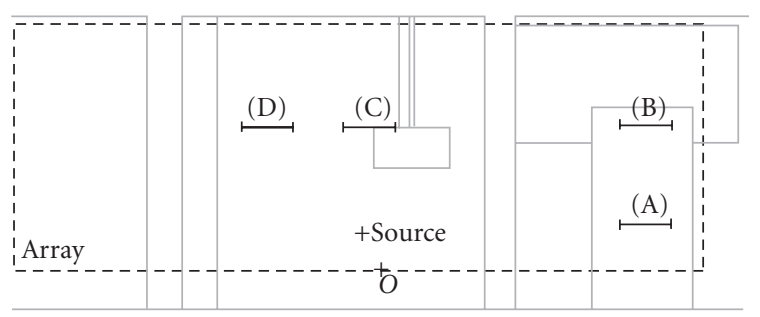

FIGURE 6: Extract of Figure 2 showing the positions of some of the cases considered.

(E) the same as (C) but with reflections from the electrical distribution box only;

(F) the direct sound at the positions of (B), (C), and (D).

Case (A) is at $0.5 \mathrm{~m}$, whereas all other cases are at $1.5 \mathrm{~m}$ above the lower array edge. Some of the positions of the different cases are shown in Figure 6. Each case consists of eleven (33 for the direct sound) ATFs from the receiver positions at $0.05 \mathrm{~m}$ intervals on a horizontal line. The ATFs of the direct sound in case (F) have been obtained directly from the original impulse response measurements. All other ATFs have been obtained from the impulse responses after demigration. Where appropriate, each case has been considered with all three wall configurations listed in Section 2.2. For example, case (C-3) refers to the ATF at the eleven positions in front of where the electrical distribution box would be, but the electrical distribution box has been removed and the wall is homogenously flat. Figure 7 shows the impulse responses used for the ATFs in cases (B), (C), and (D) in all three wall configurations (1), (2), and (3).

\subsection{Example ATFs}

Figure 8 to Figure 10 show typical magnitude and phase responses of the demigrated ATF and its model for one receiver position of case (C) in all three different wall configurations (1), (2), and (3).

What the three figures clearly show is how the complexity of the ATF is decreasing as the structural details of the reflecting wall are decreasing. The general characteristics in Figure 8 are very similar to those in Figure 9, yet the former shows larger fluctuations within small frequency bands, which is to be expected due to interference between multiple reflections. Also, in accordance with expectation is that Figure 10 exhibits the most smooth magnitude and particularly phase response of all three figures. It is also worth noting that the complexity of the ATF in the three figures is much smaller than that in Figure 5. However, the chosen order of the model is not yet sufficient to model all the details in the ATFs. This can be seen particularly well by the spurious spikes at approximately 1.2, 2.7, and $3.1 \mathrm{kHz}$ in Figure 9.

\subsection{Estimating the required model order}

The error between the ATF and its model is defined by $\epsilon(N)$. Its definition follows an output error instead of the equation 


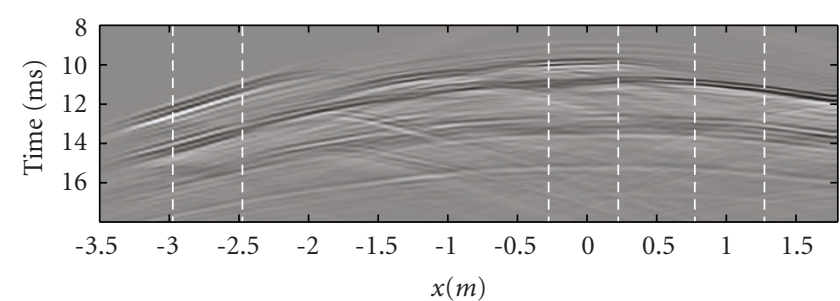

(a)

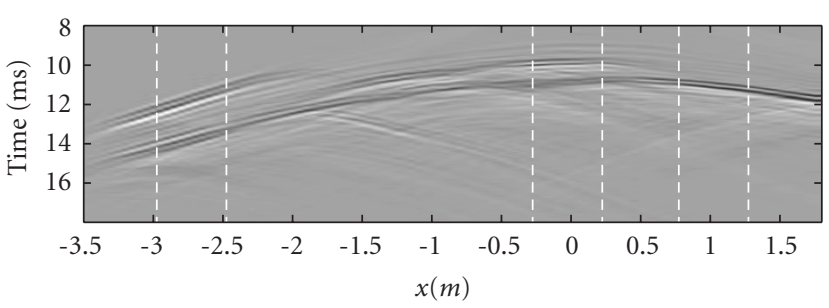

(b)

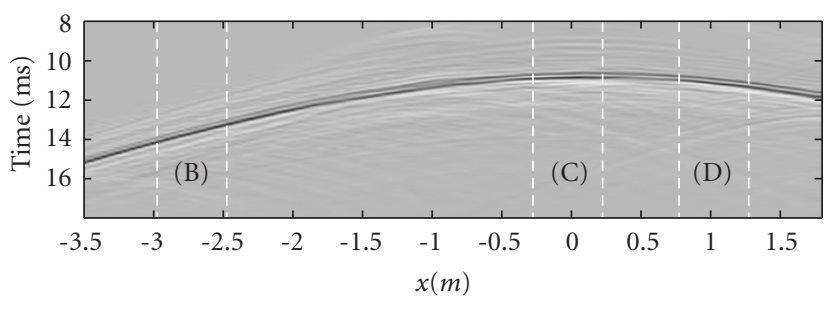

(c)

Figure 7: Demigrated impulse responses at $z=1.5 \mathrm{~m}$ used for the ATFs, (a) configuration (1), (b) configuration (2), and (c) configuration (3). The vertical lines indicate the groups of eleven impulse responses used for cases (B), (C), and (D).

error formulation of (8) and is given by

$$
\epsilon(N)=\frac{\sum_{k} w\left(\omega^{[k]}\right)\left|H\left(\omega^{[k]}\right)-B_{N}\left(\omega^{[k]}\right) / A_{N}\left(\omega^{[k]}\right)\right|^{2}}{\sum_{k} w\left(\omega^{[k]}\right)\left|H\left(\omega^{[k]}\right)\right|^{2}},
$$

with $w\left(\omega^{[k]}\right)$ defined in (9). The numerator in the above equation is the weighted sum of the squared amplitude differences between the ATF and its model and the denominator is the weighted sum of the squared magnitude of the ATF. The latter serves as a normalisation factor such that the error $\epsilon(N)$ is independent of the absolute magnitudes in the different ATFs. The required model order for a particular caseconfiguration pair is then defined as the order $N$ at which the error $\epsilon(N)$ falls below a predefined threshold. Unfortunately, defining such a threshold is inevitably a subjective matter because it depends on the desired accuracy of the model.

A practical problem that occurred when trying to find a threshold was that $\epsilon(N)$ is not necessarily a function that is monotonously decreasing before asymptotically approaching a constant value for $N$ large enough. Figure 11(a) shows $\epsilon(N)$ for all eleven ATFs of case (C-3), where it is evident that the

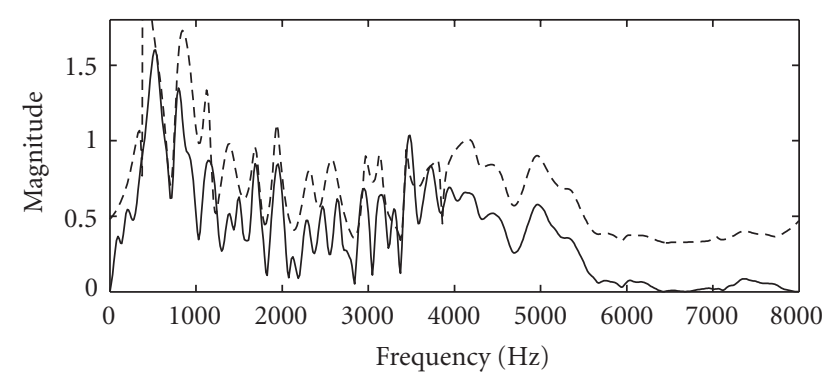

(a)

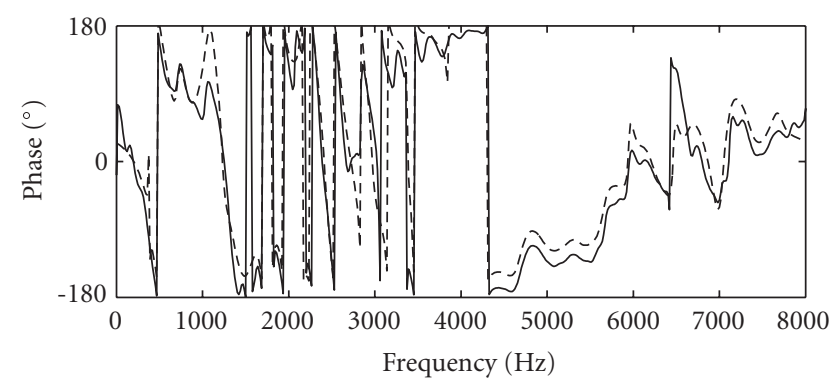

(b)

Figure 8: Case (C-1): (a) magnitude and (b) phase of ATF from demigration (solid line) and its model with $N=31$ (dashed line). Magnitude and phase of model have an offset for better comparability.

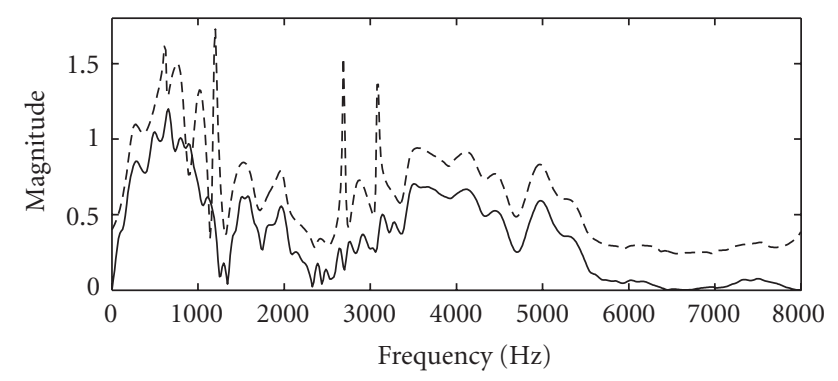

(a)

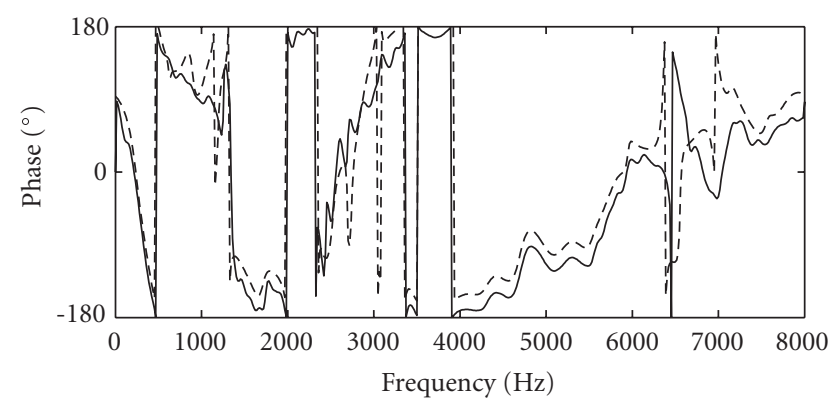

(b)

Figure 9: Case (C-2): (a) magnitude and (b) phase of ATF from demigration (solid line) and its model with $N=31$ (dashed line). Magnitude and phase of model have an offset for better comparability. 


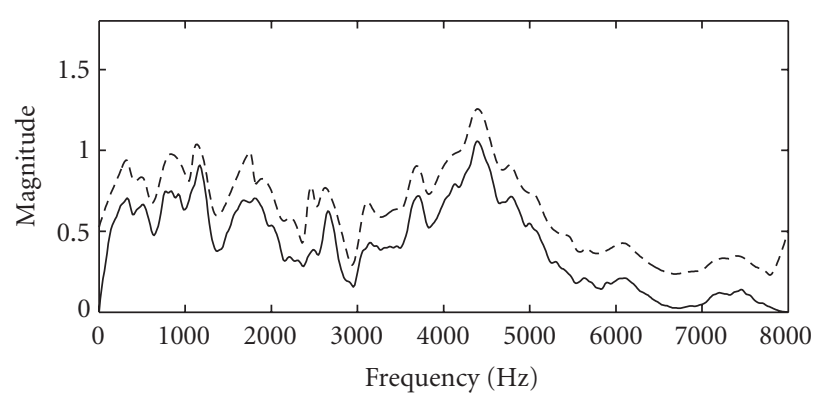

(a)

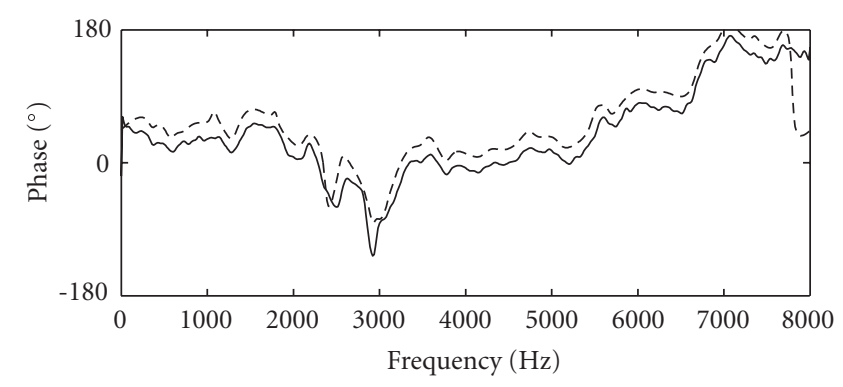

(b)

Figure 10: Case (C-3): (a) magnitude and (b) phase of ATF from demigration (solid line) and its model with $N=31$ (dashed line). Magnitude and phase of model have an offset for better comparability.

errors shoot up at discrete values of $N$. The reason for this behaviour is that the ATF model is optimum for a particular value of $N$ but there is no guarantee that a lower-order model does not have a smaller error value $\epsilon$, and further the difference between the output error in (10) and the equation error in (8) may also contribute to the problem. It is also seen in the figure that for $N$ large enough, the problem no longer occurs.

In order to circumvent these problems, a modified error function $\epsilon^{\prime}(N)$ is introduced as follows:

$$
\epsilon^{\prime}(N)=\frac{1}{N} \sum_{n=1}^{N} \epsilon(n)
$$

The modified error of the model of order $N$ is therefore the average of the errors of all models with orders from 1 to $N$. This modification is quite arbitrary but has been introduced solely to render the thresholding process more robust. It did result in a monotonously decreasing function that asymptotes a constant value for all the cases investigated. For all eleven ATFs of case (C-3), $\epsilon^{\prime}(N)$ is shown in Figure 11(b). It can be argued that a fluctuating behaviour of $\epsilon(N)$ is caused by the complexity of the ATF to be modelled, and hence the model order is too small and must be increased. Finally, the required model order was recorded as the value of $N$, where $\epsilon^{\prime}(N) \leq 0.1$. This particular value was determined experimentally and was found to (i) guarantee no visual difference

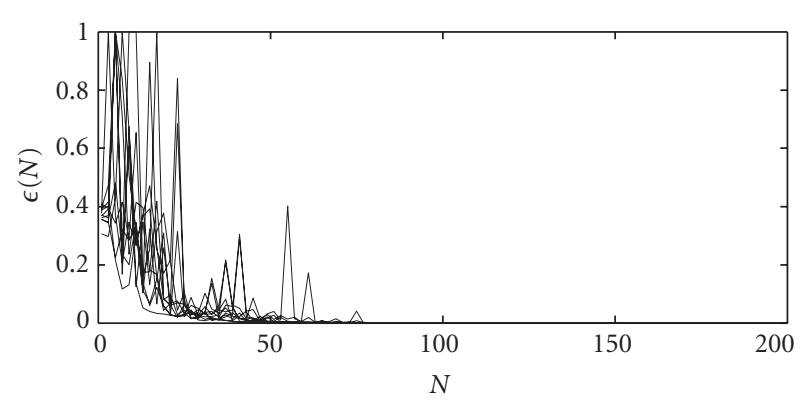

(a)

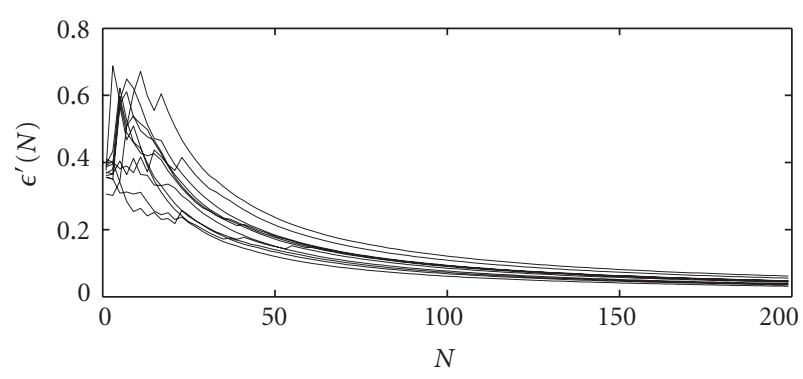

(b)

Figure 11: $\epsilon(N)$ and $\epsilon^{\prime}(N)$ for all eleven ATFs of case (C-3).

between model and actual ATF on the scale used in Figure 8 to Figure 10 and (ii) to ensure that $\epsilon(N)$ does not shoot up again for larger values of $N$ at any receiver position.

The results for the required order are expressed as mean and standard deviation between the eleven ATFs for each case and configuration and are shown in Table 1.

\section{DISCUSSION}

From Table 1, a general observation is that the highest orders for each wall configuration are mainly required for cases (B) and $(\mathrm{C})$. In fact, for both these cases, the results are very similar for wall configurations (1) and (2). For case (D), where the receivers are positioned in front of a homogenous wall section, the required order is larger in cases (D-1) and (D-2) than in cases (D-3), albeit only slightly for (D-2). This suggests that the scattering from objects such as the electrical distribution box and the columns influence the ATFs at these positions.

For wall configuration (3), that is, the homogenous flat wall, the required mean order is 83 with a standard deviation of 15 , the result for case (B-3) is almost $50 \%$ lower than for cases (C-3) and (D-3).

Another observation is that for all cases, the standard deviation of the model order depends on the wall configuration. Configuration (1) always produces the largest values and configuration (3) always the smallest. This is consistent with the fact that in the former configuration, the complexity 
TABLE 1: Mean $\bar{N}$ and standard deviation $\sigma(N)$ of the ATF model order (over the set of eleven ATFs) for the different cases and configurations.

\begin{tabular}{|c|c|c|c|c|c|c|c|c|c|c|c|c|c|}
\hline Case & $(\mathrm{A}-1)$ & $(\mathrm{A}-2)$ & (B-1) & (B-2) & $(\mathrm{B}-3)$ & $(\mathrm{C}-1)$ & $(\mathrm{C}-2)$ & $(\mathrm{C}-3)$ & (D-1) & $(\mathrm{D}-2)$ & $(\mathrm{D}-3)$ & (E) & $(\mathrm{F})$ \\
\hline $\bar{N}$ & 101 & 79 & 263 & 175 & 65 & 222 & 164 & 91 & 172 & 104 & 93 & 63 & 25 \\
\hline$\sigma(N)$ & 24 & 19 & 52 & 16 & 15 & 46 & 25 & 15 & 43 & 29 & 24 & 30 & 13 \\
\hline
\end{tabular}

of the ATF is the most position dependent because of the amount of details present.

The results for case (A) are rather inconsistent with those from the other cases. The mean order is much lower, and in particular when compared to case (B) whose receiver positions are also in front of the closet. The reason for this is currently unknown.

For case (E), which contains reflections from the electrical distribution box only, the mean order is 63. Comparing with case $(\mathrm{C}-2)$ at the same positions in front of the wall, the standard deviation is roughly the same, but the presence of the entire wall increases the mean model order to 164 .

For the direct sound (case (F)), the required order is on average 25. The figures quoted by Greendfield and Hawksford [25] for the modelling of loudspeaker transfer functions are roughly twice as large, however, due to the different methodologies employed, a direct comparison is incongruous. At any rate, the transfer function of the loudspeaker source is included in the ATFs of cases (A) to (E). In order to obtain the order of the ATF from the reflecting objects only, the order of the loudspeaker transfer function should be subtracted from the numbers listed. The lowest mean order thus obtained for a completely flat wall is 40 for case (B-3).

It is to be noted that the obtained model orders depend on the efficiency of the transfer function modelling algorithm and also on the value chosen for the error criterion $\epsilon^{\prime}$. The relative values for the model orders are therefore more meaningful and objective than the absolute values. The main findings from this paper can then be summarised as follows. The complexity (in terms of ATF model order) of sound reflections from a physical wall comprising a number of details is between two to three times higher than that of a homogenous flat wall and varies by roughly $25 \%$ between receiver positions. Further, the required model order for the physical homogenous flat wall is still relatively large. With a mirror image source model, the wall would be modelled as a single planar surface and would require one coefficient in the case of frequency-independent acoustic impedance and a slightly larger order to model the usually smooth variation with frequency. It seems very unlikely that the fluctuations in Figure 10 are only caused by acoustic impedance variations.

The applications of the acoustic imaging process, presented in [13] and the present paper, to room acoustics are the following. The method can serve as a tool to investigate sound reflection from different reflecting object (e.g., wall) details without the requirement of physically constructing the object and measuring its sound reflections in an ane- choic chamber. The results have shown that reflections from objects, whose size is in the order of the shortest acoustic wavelength, are present in the room impulse response since otherwise the object would not appear in the acoustic image. In terms of wave-equation-based room acoustic models such as finite elements, boundary elements or finite differences, the message is that leaving out objects of the size of the acoustic wavelength in question can potentially introduce significant errors, from both objective parameters and perceptual point of view. An example is the increased "diffusion" offered by the object details as compared to larger planar surfaces. It is conceivable that the presented method can be extended to quantify these errors objectively.

For room acoustic models based on geometrical acoustics, the results from the macroscopic flat wall with frequency-dependent reflectivity properties reinforce the importance of incorporating and assigning a nonzero value for the diffusion/scattering coefficient even to planar surfaces. Further, comparing the complexity of the ATF from the geometrical acoustics model with that from the acoustic image can aid in the task of accurately modelling diffraction and scattering through surface and edge sources. This in turn can potentially help to improve the physical accuracy versus computational complexity dilemma encountered in room acoustics modelling. A further interesting avenue is the perceptual comparison between the reflections from the macroscopic flat wall in this paper and the flat wall from a geometrical acoustics model.

\section{CONCLUSION}

The modelling of acoustic transfer function (ATF) from the reflections of single objects has been performed. The purpose was to investigate the complexity of the reflections from real physical objects. The ATFs have been obtained by demigrating the acoustic image of the reflecting objects, consisting of a corridor wall with a number of details such as columns, a closet, and an electrical distribution box. The original acoustic image has been augmented first by simplifying it and then by replacing larger objects with homogenous wall sections.

The ATFs to be modelled stem from various receiver positions in front of the wall. The required model order has been estimated from the error between modelled and actual ATFs. It was found that the maximum and minimum total model orders $2 N$ are 526 and 126, respectively. For the ATF from the original unaltered acoustic image, the variation in model order as a function of receiver positions is the largest and approximately $25 \%$. The lowest model order 
for the homogenous flat wall was 130. Finally, the order $2 N$ of the loudspeaker transfer function, which is implicitly included in the ATF, was estimated as 50. This figure would need to be subtracted from the above-quoted numbers.

The results in this paper confirmed the applicability of some of the practices in current room acoustics modelling and the method itself can be used to further understand and improve the modelling of reflections from real physical objects.

\section{ACKNOWLEDGMENTS}

The authors thank three anonymous reviewers and the Associate Editor Aki Härmä for valuable remarks about the manuscript text.

\section{REFERENCES}

[1] J. B. Allen and D. A. Berkley, "Image method for efficiently simulating small-room acoustics," Journal of the Acoustical Society of America, vol. 65, no. 4, pp. 943-950, 1979.

[2] H. Kuttruff, Room Acoustics, chapter 2, Spon Press, London, UK, 4th edition, 2000.

[3] M. Vorländer, "Simulation of the transient and steadystate sound propagation in rooms using a new combined ray-tracing/image-source algorithm," Journal of the Acoustical Society of America, vol. 86, no. 1, pp. 172-178, 1989.

[4] I. A. Drumm and Y. W. Lam, "The adaptive beam-tracing algorithm," Journal of the Acoustical Society of America, vol. 107, no. 3, pp. 1405-1412, 2000.

[5] A. J. Berkhout, D. de Vries, J. Baan, and B. W. van den Oetelaar, "A wave field extrapolation approach to acoustical modeling in enclosed spaces," Journal of the Acoustical Society of America, vol. 105, no. 3, pp. 1725-1733, 1999.

[6] B.-I. L. Dalenbäck, "Room acoustic prediction based on a unified treatment of diffuse and specular reflection," Journal of the Acoustical Society of America, vol. 100, no. 2, pp. 899-909, 1996.

[7] B.-I. L. Dalenbäck, "Verification of prediction based on randomized tail-corrected cone-tracing and array modeling," in Proceedings of the 137th ASA Meeting, 2nd Convention of the European Acoustics Association and 25th German Acoustics DAGA Conference, Berlin, Germany, March 1999.

[8] J. J. Embrechts, "Broad spectrum diffusion model for room acoustics ray-tracing algorithms," Journal of the Acoustical Society of America, vol. 107, no. 4, pp. 2068-2081, 2000.

[9] Y. W. Lam, "A comparison of three diffuse reflection modeling methods used in room acoustics computer models," Journal of the Acoustical Society of America, vol. 100, no. 4, pp. 21812192, 1996.

[10] R. R. Torres, U. P. Svensson, and M. Kleiner, "Computation of edge diffraction for more accurate room acoustics auralization," Journal of the Acoustical Society of America, vol. 109, no. 2, pp. 600-610, 2001.

[11] U. P. Svensson, R. I. Fred, and J. Vanderkooy, "An analytic secondary source model of edge diffraction impulse responses," Journal of the Acoustical Society of America, vol. 106, no. 5, pp. 2331-2344, 1999.

[12] A. Farina, "Introducing the surface diffusion and edge scattering in a pyramid-tracing numerical model for room acous- tics," in Proceedings of the 108th Audio Engineering Society Convention (AES 'O0), Paris, France, February 2000.

[13] M. Kuster, D. de Vries, E. M. Hulsebos, and A. Gisolf, "Acoustic imaging in enclosed spaces: analysis of room, geometry modifications on the impulse response," Journal of the Acoustical Society of America, vol. 116, no. 4, pp. 2126-2137, 2004.

[14] D. de Vries, N. Joeman, and E. Schreurs, "Measurement-based simulation and analysis of scattering structures," in Proceedings of the Forum Acusticum, Budapest, Hungary, August 2005.

[15] D. J. Verschuur, private communication, 2006.

[16] M. Tygel, J. Schleicher, and P. Hubral, "A unified approach to 3-D seismic reflection imaging, part II: theory," Geophysics, vol. 61, no. 3, pp. 759-775, 1996.

[17] E. W. Start, V. G. Valstar, and D. de Vries, "Application of spatial bandwidth reduction in wave field synthesis," in Proceedings of the 98th Audio Engineering Society Convention, Paris, France, February 1995.

[18] D. de Vries, M. Kuster, and E. Hulsebos, "Analyzing the influence of design modification on the room response by acoustical imaging," in Proceedings of the International Symposium on Room Acoustics: Design and Science (RADS '04), Hyogo, Japan, April 2004.

[19] E. C. Levy, “Complex-curve fitting," IRE Transactions on Automatic Control, vol. 4, no. 3, pp. 37-44, 1959.

[20] Y. Haneda, S. Makino, and Y. Kaneda, "Common acoustical pole and zero modeling of room transfer functions," IEEE Transactions on Speech and Audio Processing, vol. 2, no. 2, pp. 320-328, 1994.

[21] J. Mourjopoulos and M. A. Paraskevas, "Pole and zero modeling of room transfer functions," Journal of Sound and Vibration, vol. 146, no. 2, pp. 281-302, 1991.

[22] M. Karjalainen, T. Paatero, J. N. Mourjopoulos, and P. D. Hatziantoniou, "About room response equalization and dereverberation," in Proceedings of IEEE Workshop on Applications of Signal Processing to Audio and Acoustics (WASPAA '05), pp. 183-186, New Paltz, NY, USA, October 2005.

[23] M. R. Schroeder, "Statistical parameters of the frequency response curves of large rooms," Journal of the Audio Engineering Society, vol. 35, no. 5, pp. 299-306, 1987.

[24] M. Schönle, U. Zölzer, and M. Fliege, "Modeling of room impulse responses by multirate systems," in Proceedings of the 93rd Audio Engineering Society Convention, San Francisco, Calif, USA, October 1992.

[25] R. Greenfield and M. O. Hawksford, "Efficient filter design for loudspeaker equalization," Journal of the Audio Engineering Society, vol. 39, no. 10, pp. 739-751, 1991.

Martin Kuster obtained a B.Eng. degree in electroacoustics from the University of Salford, UK, in 2001 and an M.S. degree in applied physics from Delft University of Technology, The Netherlands, in 2003. He joined the Queen's University Belfast in September 2004 to work on a collaborative project with a digital entertainment company, in the framework of which he undertakes research into room acoustic (inverse) mod-

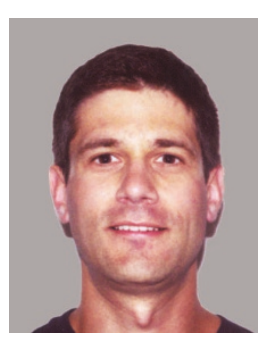

elling. Previously, he has been researching the use of acoustic imaging in room acoustics and bending wave transducer technology in the context of wave-field synthesis. He is a Member of the Acoustical Society of America and the Audio Engineering Society. 
Diemer de Vries was born January 3, 1945, in Weststellingwerf, Friesland, The Netherlands. He received his M.S. degree from Delft University of Technology in 1971 on a thesis on architectural acoustics. During his career as a University Researcher, he worked on several projects in room acoustics, building acoustics, and seismic signal processing. In 1984, he received a Ph.D. degree on a thesis in the latter field. He now coordi-

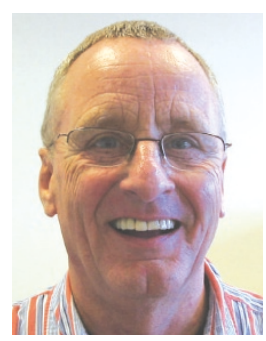
nates, as an Associate Professor, the research on array technologybased wave-field analysis and synthesis in room acoustics. By participating with his group in national and international projects (such as CARROUSO, 2001-2003), he significantly contributed to the breakthrough of this concept-introduced by Berkhout in the 1980s-all over the world. He has great interest in scientific education. He gave and gives many courses on acoustics, building physics, and general physics, at the university and beyond. He is Past Chairman of the Dutch Acoustical Association (Nederlands Akoestisch Genootschap, NAG) and of the Netherlands Section of the Audio Engineering Society (AES) which gave him a Certificate of Appreciation in 1995. In 1999, the international AES board gave him a Fellowship Award for his contributions to the implementation of wave-field synthesis. 